\title{
Primary pleural epithelioid sarcoma of the proximal type: a diagnostic and therapeutic challenge
}

\author{
Zeeshan Ahmad ${ }^{1}$, Qasim Stanazai ${ }^{2}$, Staphanie Wright ${ }^{3}$, Matthew Smolkin ${ }^{3}$, Patrick C. Ma \\ ${ }^{1}$ Section of Hematology/Oncology, Department of Medicine, West Virginia University School of Medicine, Morgantown, WV, USA; ${ }^{2}$ Department \\ of Medicine, West Virginia University School of Medicine, Morgantown, WV, USA; ${ }^{3}$ Section of Molecular Pathology, Department of Pathology, \\ West Virginia University School of Medicine, Morgantown, WV, USA; ${ }^{4}$ Penn State Cancer Institute, Penn State Health Milton S. Hershey Medical \\ Center, Pennsylvania State University, Hershey, PA, USA \\ Correspondence to: Patrick C. Ma, MD, MSc. Professor of Medicine, Associate Director of Translational Research, Thoracic Oncology Disease Team \\ Leader, Penn State Cancer Institute, Penn State Health Milton S. Hershey Medical Center, Pennsylvania State University, 500 University Drive, \\ Hershey, PA 17033, USA. Email: patrickma@pennstatehealth.psu.edu.
}

\begin{abstract}
Epithelioid sarcoma (ES) is an uncommon soft tissue neoplasm first described in 1970. It is a unique soft tissue neoplasm of adolescents and younger adults which usually presents as a subcutaneous and deep dermal mass in the distal portions of the extremities. The proximal-type variant of this rare soft tissue neoplasm was only recently reported. The proximal form typically arise in proximal extremities and in the deep parts of pelvis, perineum and genital tract. The proximal type variant has distinct histological characteristics and aggressive clinical course as compared to the distal ES. Inactivation of INI1 has been reported in both distal and proximal variants and can help to make the diagnosis. Furthermore, the proximal variant has a possible association with malignant rhabdoid neoplasm. We describe here a case of primary pleural ES of the proximal type and highlight its diagnostic and therapeutic challenges.
\end{abstract}

Keywords: Epithelioid sarcoma (ES); proximal type; primary pleural; rare sarcoma

Submitted Apr 05, 2019. Accepted for publication Sep 09, 2019.

doi: $10.21037 /$ tlcr.2019.09.16

View this article at: http://dx.doi.org/10.21037/tlcr.2019.09.16

\section{Introduction}

Epithelioid sarcoma (ES) is an uncommon soft tissue neoplasm first described in 1970 (1). It is a unique soft tissue neoplasm of adolescents and younger adults which usually presents as a subcutaneous and deep dermal mass in the distal portions of the extremities (2). The proximal-type variant of this rare soft tissue neoplasm was only recently reported (3). The proximal form typically arise in proximal extremities and in the deep parts of pelvis, perineum and genital tract (3). The proximal type variant has distinct histological characteristics and aggressive clinical course as compared to the distal ES $(3,4)$. Inactivation of SMARCB1 (also known as BAF47, INI1) has been reported in both distal and proximal variants and can help to confirm the diagnosis (5). Furthermore, the proximal variant has a possible association with malignant rhabdoid neoplasm (6). We describe here a case of primary pleural ES of the proximal type and highlight its diagnostic and therapeutic challenges.

\section{Case presentation}

A 27-year-old Caucasian never-smoker male with no significant past medical history, developed cough and SOB for almost a month in September 2017 while working in Texas (TX). He worked on natural gas rigs and had no history of asbestos exposure. He sought medical attention, and was diagnosed with pneumonia. Oral antibiotics were started which did not resolve his symptoms. His dyspnea continues to worsen and he returned to the hospital where a CT scan of chest revealed a large left pleural effusion and multiple hypermetabolic pleural nodules confirmed with a positron emission tomography-computed tomography (PET/CT) scan imaging (Figure 1). He underwent videoassisted thoracoscopy (VATS) with drainage of the pleural 

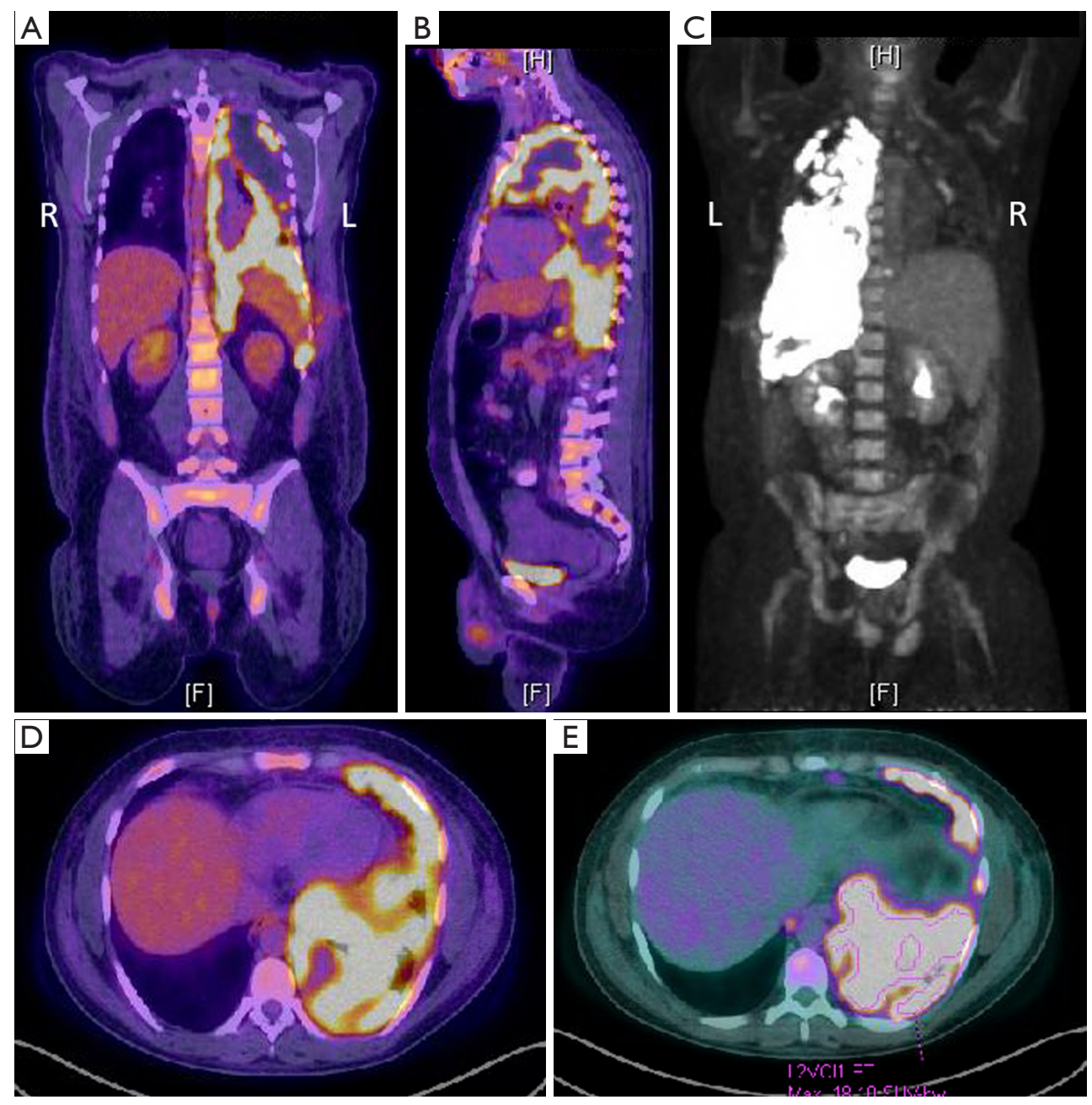

Figure 1 Radiographic scan imaging results of the primary pleural epithelioid sarcoma of the proximal type. PET/CT scan imaging showing increased 18F-fluorodeoxyglucose uptake in the left hemithorax and lymph nodes in the mediastinal and periaortic regions. (A) Coronal view of PET/CT overlay image; (B) lateral view of PET/CT overlay image; (C) coronal view of the PET image; (D) cross-sectional view of the PET/CT overlay image; (E) cross-sectional view of the PET/CT overlay image. PET/CT, positron emission tomography-computed tomography.

effusion. Biopsies of the pleural nodules were performed during VATS with the resultant pathology somewhat non-specific, and reported as "poorly differentiated" malignancy and was concerning for a "primary pleural ES of proximal type". Mesothelioma was considered to be less likely. The histologic sections (H\&E) demonstrated sheets of pleomorphic tumor cells with epithelioid features (200x) (Figure 2A). The specimens were also sent to M.D. Anderson Cancer Center for a second opinion, which returned as consistent with "poorly differentiated highgrade epithelioid malignancy. The immunoreactivity profile (Figure 2B, C,D,E) was found as follows: CK7 and CK20 both negative, but positive for pancytokeratin, CD34, WT1 , calretinin (very focally). There was also loss of SMARCB1 (BAF47, INI-1). Additional immunoreactivity was negative for CK5/6, TTF-1, D2-40, Napsin-A, p63, p40, MOC31, Ber-EP4, S100, CD30, CD31, SOX10, desmin and ERG, raising the possibility of a "primary pleural ES of the proximal type". There was heterogeneous deletion of $C D K N 2 A$ (P16) by fluorescence in situ hybridization (FISH) which was deemed to relatively argue against the possibility of mesothelioma. Comprehensive tumor molecular profiling through Caris Molecular Intelligence (Caris Life Sciences, Phoenix, AZ, USA) profiling revealed 

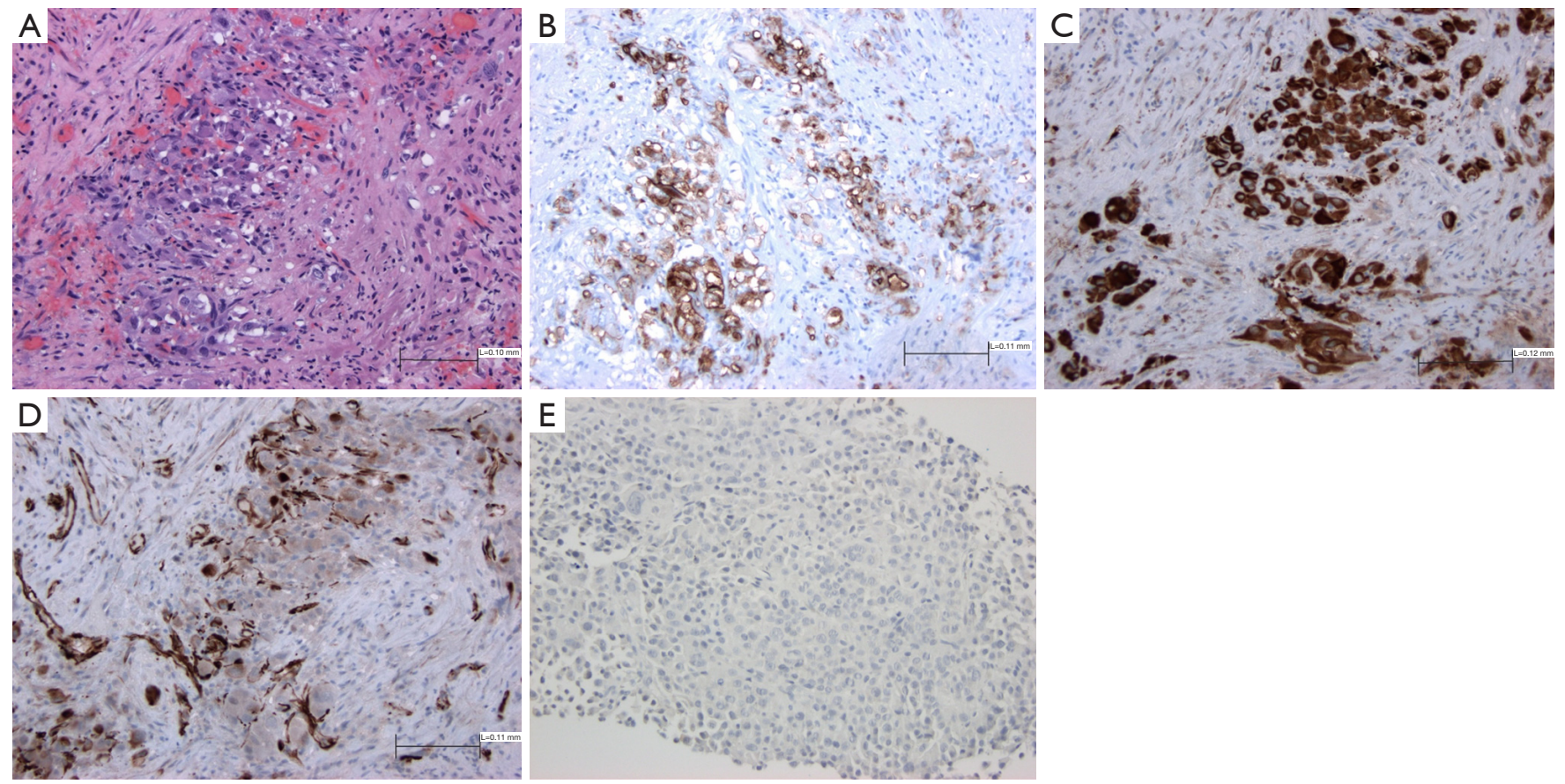

Figure 2 Histologic findings of the primary pleural epithelioid sarcoma of the proximal type. (A) H\&E staining demonstrated the characteristic epithelioid or polygonal tumor cells. 200x; (B) CD34: An immunohistochemical stain for CD34 highlighted moderate to strong membranous and cytoplasmic staining of tumor cells, 200×; (C) AE1/AE3: An IHC stain for AE1/AE3 highlighted strong cytoplasmic staining of tumor cells, 200×. (D) WT-1: An immunohistochemical stain for WT-1 showed positive cytoplasmic staining of tumor cells, 200×; (E) PD-L1 (22C3): An immunohistochemical stain for PD-L1 was found to be negative (TPS 0\%), 200×. PD-L1, programmed deathligand 1.

no readily actionable molecular-genomic lesion and the immune checkpoint pathway biomarker programmed death-ligand 1 (PD-L1) expression was found to be negative (TPS 0\%) (Tables 1-4, Figures 2E,S1,S2). A pathogenic SMARCB1 (BAF47, INI-1) I125fs (exon 6) frameshift mutation was identified. Tumor mutational load (TML) was found to be intermediate at 7 mutations/megabase, and the microsatellite instability (MSI) status was stable. Of note, a presumed benign BRCA2 T3013I mutation (exon 23) was found while $B R C A 1$ was non-mutated.

After the initial presentation and diagnostic workup, patient returned to West Virginia (WV) to be closer to his families for further oncologic care and treatment. His final diagnosis was determined in a multidisciplinary thoracic oncology tumor board conference to be advanced metastatic primary pleural ES of proximal type cT4N1M1, Stage IV (AJCC TNM eighth edition, 2017) with extensive left pleural thickening and nodularity, malignant left pleural effusion, and also metastases to mediastinal and paraaortic lymph nodes. He was also found to have bone metastasis with pathologic fracture of his left humerus. He was initially started on first-line combination chemotherapy with the MAI regimen [doxorubicin $25 \mathrm{mg} / \mathrm{m}^{2} \times 3$ days intravenous (IV) continuous infusion, ifosfamide with $25 \%$ dose reduction at $1,875 \mathrm{mg} / \mathrm{m}^{2} \times$ days $\mathrm{IV}$ and with mesna support].

His hospitalization was complicated with acute renal insufficiency requiring continuous renal replacement therapy (CRRT), sepsis with candidemia, systemic inflammatory response syndrome (SIRS) and respiratory failure. Moreover, patient developed acute mental status change which was attributed to metabolic derangement from multi-organ dysfunction, poor respiratory function or chemotherapy-induced neurotoxicity. Ifosfamide was held and patient was started on methylene blue. Subsequently patient was transferred to medical intensive care unit (ICU) for acute hypoxic respiratory failure requiring mechanical ventilation. Patient underwent flexible bronchoscopy, limited left thoracotomy, left pleural mass biopsy, and placement of left thoracotomy tube for large sanguineous pleural effusion. Frozen section of pleural mass was 
Table 1 Summary of the notable biomarkers findings from the Caris MI profile

\begin{tabular}{|c|c|c|}
\hline Biomarker & Method & Results \\
\hline Total mutational load & NGS & $\begin{array}{l}\text { Intermediate/7 } \\
\text { mutations/Mb }\end{array}$ \\
\hline MSI & NGS & Stable \\
\hline PD-L1 & $\mathrm{IHC}$ & Negative/0, 100\% \\
\hline \multirow[t]{2}{*}{ SMARCB1 } & NGS & Mutated, Pathogenic \\
\hline & & Exon $6 / 1245 f s$ \\
\hline ATM & NGS & Mutation not detected \\
\hline BRAF & NGS & Mutation not detected \\
\hline BRCA1 & NGS & Mutation not detected \\
\hline \multirow[t]{2}{*}{ BRCA2 } & NGS & $\begin{array}{l}\text { Mutated, presumed } \\
\text { benign }\end{array}$ \\
\hline & & Exon 23/T3013I \\
\hline EGFR & NGS & Mutation not detected \\
\hline KRAS & NGS & Mutation not detecteo \\
\hline NRAS & NGS & Mutation not detected \\
\hline HER2/NEU (ERBB2) & NGS & $\begin{array}{l}\text { Amplification not } \\
\text { detected }\end{array}$ \\
\hline ERCC1 & $\mathrm{IHC}$ & Negative/0, 100\% \\
\hline RRM1 & $\mathrm{IHC}$ & Negative/2+, $35 \%$ \\
\hline TS & $\mathrm{IHC}$ & Positive, 1+, 10\% \\
\hline TUBB3 & $\mathrm{IHC}$ & Positive/2+, 90\% \\
\hline
\end{tabular}

MSI, microsatellite instability; NGS, next-gene sequencing; PDL1, programmed death-ligand 1; IHC, immunohistochemistry.

consistent with epithelioid neoplasm. Bronchoscopy was remarkable only for extrinsic compression of the left lower lobe bronchus. Consensus decision was then reached for patient to be extubated with no plan for reintubation. His clinical condition rapidly deteriorated thereafter post compassionate terminal extubation and he subsequently expired soon after in the ICU while on comfort care, about 2 months after his initial diagnosis.

\section{Discussion}

Proximal epithelioid sarcoma (PES) usually involves males in their early decades of life (7). PES has been reported frequently in the extremities but other locations including palate, penis, perineum and the scalp have been reported in the literature too (8). Primary pleural ES of the proximal type is quite rare, and to the best of our knowledge, there are only 2 prior published case reports in the literature.

ES can be histologically categorized into classical, spindle and mixed forms. Furthermore, the tumor can be classified as a proximal or axial types based on their location. Based on the reported literature apparently the PESs have the worse outcomes. Histological diagnosis could be challenging at times due to shared histological features with other neoplasms including extrarenal rhabdoid sarcomas, clear cell sarcoma and squamous cell carcinoma. Due to the same histological mimicry ES need to be differentiated from inflammatory conditions like nodular fasciitis and tenosynovitis as well as from granulomatous processes (7).

The distinction of proximal-type ES from undifferentiated carcinoma is one of the most difficult consideration. The presence of CD34 reactivity, the occurrence of tumors in deep soft tissues without any connection with the overlying epidermis or cutaneous adnexal tissue and the absence of histologic features of squamous or glandular differentiation favor the diagnosis of ES over undifferentiated carcinoma (2).

Given the fact that malignant as well as benign vascular endothelial cells may express keratins, differential immunostaining should include anti-CD31. CD31 typically shows a distinctive membrane pattern of reactivity in vascular tumors that is typically not encountered in ESs (9). Epithelioid angiosarcomas may be cytokeratin positive, but they are usually positive for endothelial markers, such as CD34 and CD31, whereas ESs can display positivity for CD34, but are negative for CD31 $(9,10)$.

FISH analysis of primary malignant mesothelioma tissue samples or malignant mesothelioma cells from the pleural effusion usually show homozygous deletions of the $C D K N 2 A / A R F$ locus in almost $70 \%$ of the cases $(11,12)$. Based on histological subclassification, malignant mesothelioma of epithelioid variety shows almost $70 \%$ of homozygous deletion of $C D K N 2 A$ and sarcomatoid type reveals around $100 \%$ of homozygous deletion (13). In general, malignant mesotheliomas display homozygous deletion of $C D K N 2 A$, whereas homozygous deletions are usually not found in sarcomas (14). In fact, SMARCB1 (BAF47, INI1) abnormalities have the highest rate of recurrence in ES patients. Consequently, the identification of the tumor suppressor gene SMARCB1 inactivation also favors the diagnosis of proximal-type ES (15). SMARCB1 is a known tumor suppressor gene implicated in cell growth and development, and is also known as SWI/SNF related, matrix associated, actin dependent regulator of chromatin, 
Table 2 Summary of the detected genomic mutations of SMARCA1 and BRAC2

\begin{tabular}{lccccc}
\hline Gene & Method & Result & Alteration & Frequency (\%) & Exon \\
\hline BRCA2 & NGS & Mutated, Presumed Benign & T3013| & 49 & 23 \\
SMARCB1 & NGS & Mutated, Pathogenic & I245fs & 20 & 6 \\
\hline
\end{tabular}

NGS, next-gene sequencing.

Table 3 Summary of the IHC biomarkers results

\begin{tabular}{lc}
\hline Biomarker & Result \\
\hline ERCC1 & Negative/0, 100\% \\
PD-L1 & Negative/0, 100\% \\
TS & Positive/1+, 10\% \\
RRM1 & Negative/2+, 35\% \\
TOP2A & Positive/2+, 10\% \\
TUBB3 & Positive/2+, 90\% \\
\hline
\end{tabular}

IHC, Immunohistochemistry; PD-L1, programmed death-ligand 1.

Table 4 Genes tested with indeterminate sequencing results by NGS

Genes
ATRX
KDM5C
KDM6A
KMT2C
RAD50
SMARCE1

NGS, next-gene sequencing.

subfamily b, member 1 . Inactivating loss of expression of SMARCB1 has been reported in tumors including ES, and also renal medullary carcinoma, undifferentiated pediatric sarcomas, and a subset of hepatoblastomas. Germline mutations in SMARCB1 have also been found to cause $\sim 20 \%$ of rhabdoid tumors, and a subset of schwannoma and meningioma. The morphological characteristics, immunohistochemistry, and molecular phenotype of our case were consistent with ES of proximal type, rather than mesothelioma, carcinoma or angiosarcoma.

An accurate diagnosis is imperative to choose the optimal treatment and obtain the best outcome for the patient. In the clinical practice this will influence the course of treatment. Malignant pleural mesothelioma and sarcomas do not respond to the same treatment modalities. While cisplatin and pemetrexed are considered the first-line treatment regimen for malignant pleural mesothelioma, doxorubicin is the first-line modality for treating proximaltype ESs. Furthermore, second and third line treatment options also differ (16).

\section{Conclusions}

Proximal-type ESs are extremely rare and are notorious for high local recurrence rates and distant metastasis in up to $60 \%$ of cases $(17,18)$. These tumors, when arising in proximal locations, have a very poor prognosis than those arising in distal locations. Despite surgical resection, patients with early tumor metastasis and large masses are associated with poor overall outcome. The lack of directed therapies against proximal-type ES highlights the need to determine the underlying molecular causes of the disease. Comprehensive tumor molecular profiling should be considered to better understand the molecular drivers and guide more precise and individualized treatment decisions.

\section{Acknowledgments}

We thank our patient in participating in our study. This study was supported by WVU Cancer Institute, West Virginia University and Penn State Cancer Institute, Penn State Health Milton S. Hershey Medical Center.

\section{Footnote}

Conflicts of Interest: The authors have no conflicts of interest to declare.

Ethical Statement: The authors are accountable for all aspects of the work in ensuring that questions related to the accuracy or integrity of any part of the work are appropriately investigated and resolved. Written informed consent (IRB approved study protocol WVU011117) was obtained from the patient for publication of this case report and any accompanying images. 


\section{References}

1. Enzinger FM. Epitheloid sarcoma. A sarcoma simulating a granuloma or a carcinoma. Cancer 1970;26:1029-41.

2. Hasegawa T, Matsuno Y, Shimoda T, et al. Proximal-type epithelioid sarcoma: a clinicopathologic study of 20 cases. Mod Pathol 2001;14:655-63.

3. Guillou L, Wadden C, Coindre JM, et al. "Proximaltype" epithelioid sarcoma, a distinctive aggressive neoplasm showing rhabdoid features. Clinicopathologic, immunohistochemical, and ultrastructural study of a series. Am J Surg Pathol 1997;21:130-46.

4. Naik D, Kumar AA, Srinath MG. Proximal-type epithelioid sarcoma-a rare soft tissue sarcoma of thigh in a child. Indian J Surg Oncol 2011;2:298-301.

5. Hornick JL, Dal Cin P, Fletcher CD. Loss of INI1 expression is characteristic of both conventional and proximal-type epithelioid sarcoma. Am J Surg Pathol 2009;33:542-50.

6. Chbani L, Guillou L, Terrier P, et al. Epithelioid sarcoma: a clinicopathologic and immunohistochemical analysis of 106 cases from the French sarcoma group. Am J Clin Pathol 2009;131:222-7.

7. Saha D, Basu A, Maiti A, et al. Primary proximal epithelioid sarcoma of the lung successfully treated with pneumonectomy and adjuvant chemotherapy. BMJ Case Rep 2016;2016.

8. Etienne-Mastroianni B, Falchero L, Chalabreysse L, et al. Primary sarcomas of the lung: a clinicopathologic study of 12 cases. Lung Cancer 2002;38:283-9.

9. Miettinen M, Fanburg-Smith JC, Virolainen M, et al.

Cite this article as: Ahmad Z, Stanazai Q, Wright S, Smolkin M, Ma PC. Primary pleural epithelioid sarcoma of the proximal type: a diagnostic and therapeutic challenge. Transl Lung Cancer Res 2019;8(5):700-705. doi: 10.21037/tlcr.2019.09.16
Epithelioid sarcoma: an immunohistochemical analysis of 112 classical and variant cases and a discussion of the differential diagnosis. Hum Pathol 1999;30:934-42.

10. Armah HB, Parwani AV. Epithelioid sarcoma. Arch Pathol Lab Med 2009;133:814-9.

11. Chiosea S, Krasinskas A, Cagle PT, et al. Diagnostic importance of 9p21 homozygous deletion in malignant mesotheliomas. Mod Pathol 2008;21:742-7.

12. Illei PB, Rusch VW, Zakowski MF, et al. Homozygous deletion of CDKN2A and codeletion of the methylthioadenosine phosphorylase gene in the majority of pleural mesotheliomas. Clin Cancer Res 2003;9:2108-13.

13. Sekido Y. Molecular pathogenesis of malignant mesothelioma. Carcinogenesis 2013;34:1413-9.

14. Tochigi N, Attanoos R, Chirieac LR, et al. p16 Deletion in sarcomatoid tumors of the lung and pleura. Arch Pathol Lab Med 2013;137:632-6.

15. Modena P, Lualdi E, Facchinetti F, et al. SMARCB1/ INI1 tumor suppressor gene is frequently inactivated in epithelioid sarcomas. Cancer Res 2005;65:4012-9.

16. Madsen GA, Rasmussen TR, Baerentzen S. A case of pleural epithelioid sarcoma of proximal type presenting as malignant pleural mesothelioma. J Thorac Oncol 2013;8:e89-90.

17. Echchaoui A, Sadrati Y, Elbir Y, et al. Proximal-type epithelioid sarcoma: a new case report and literature review. Pan Afr Med J 2016;24:238.

18. Casanova M, Ferrari A, Collini P, et al. Epithelioid sarcoma in children and adolescents: a report from the Italian Soft Tissue Sarcoma Committee. Cancer 2006;106:708-17. 
Supplementary

\begin{tabular}{|c|c|c|c|c|c|c|c|c|c|c|c|}
\hline$A B L 1$ & $A K T 1$ & $A L K$ & AMER1 & $A P C$ & $A R$ & $A R A F$ & ARID1A & ARID2 & ATM & $B A P 1$ & $B L M$ \\
\hline CDKN1B & CDKN2A & CHEK1 & CHEK2 & $C I C$ & $C-M E T$ & CSF1R & CTNNB1 & $D D R 2$ & DICER1 & EGFR & ERBB3 \\
\hline ERBB4 & ERCC2 & ESR1 & FANCC & FBXW7 & FGFR1 & FGFR2 & FGFR3 & FGFR4 & $F H$ & FLCN & FLT1 \\
\hline FLT3 & FLT4 & FOXL2 & FUBP1 & GATA3 & GNA11 & $G N A Q$ & GNAS & $\begin{array}{c}\text { HER2 } \\
\text { (ERBB2) }\end{array}$ & HIST1H3B & HNF1A & HRAS \\
\hline MEK2 & MEN1 & MITF & MLH1 & $M P L$ & MRE11A & MSH2 & MSH6 & MTOR & MUTYH & $N B N$ & $N F 1$ \\
\hline NF2 & NOTCH1 & NPM1 & NRAS & NTRK1 & NTRK2 & NTRK3 & PALB2 & PBRM1 & PDGFRA & PDGFRB & PHOX2B \\
\hline PIКЗСA & PIK3R1 & PMS1 & PMS2 & POLE & POT1 & PPARG & $P P P 2 R 1 A$ & PRKAR1A & PRKDC & PTCH1 & PTEN \\
\hline$V H L$ & WRN & WT1 & & & & & & & & & \\
\hline
\end{tabular}

Figure S1 Summary of the Caris MI profiling results of the genes without point mutations or indels genomic alterations by NGS. MI, molecular intelligence; NGS, next-gene sequencing.

\begin{tabular}{|l|c|c|c|c|c|c|c|c|c|c|c|}
\hline AKT2 & ALK & ARID1A & AURKB & CCND1 & CCND3 & CCNE1 & $\begin{array}{c}\text { CD274 } \\
\text { (PD-L1) }\end{array}$ & CDK4 & CDK6 & CDK8 & CDKN2A \\
\hline C-MET & CREBBP & CRKL & EGFR & EP300 & EZH2 & FGF10 & FGF3 & FGF4 & FGFR1 & FGFR2 & FGFR3 \\
\hline GATA3 & $\begin{array}{c}\text { HER2 } \\
\text { (ERBB2) }\end{array}$ & $\begin{array}{c}\text { KDR } \\
\text { (VEGFR2) }\end{array}$ & MCL1 & MDM2 & MEK1 & MYC & NF2 & NFKB1A & NTRK1 & RB1 & RICTOR \\
\hline ROS1 & TOP1 & WT1 & & & & & & & & & \\
\hline
\end{tabular}

Figure S2 Genes tested without copy number variations (amplifications) by NGS. NGS, next-gene sequencing. 